\title{
Dynamic Micromagnetic Simulation of the Configurational Anisotropy of Nanoelements
}

\author{
Josef Fidler, Thomas Schrefl, Dieter Süss, and Werner Scholz
}

\begin{abstract}
A finite element method was used to simulate the magnetization reversal of nanostructured $\mathrm{Ni}_{80} \mathrm{Fe}_{20}$ elements with zero anisotropy. The numerical results show a strong influence of the size of the cubic and platelet shaped (square and triangular) elements on the switching field. The calculated switching fields range from $\mu_{0} I=0.002$ to $0.6 \mathrm{~T}$. Differences of the demagnetizing field which arise when the field is applied in different directions, lead to configurational anisotropy effects. Platelet shaped elements show identical switching behavior in different directions within the platelet plane. Inhomogeneous magnetization reversal processes become dominant with increasing element size $\geq 100 \mathrm{~nm}$ and strongly influence the switching behavior.
\end{abstract}

Index Terms-Configurational anisotropy, nanomagnets, numerical micromagnetics, switching field.

\section{INTRODUCTION}

$\mathbf{O}$ VER RECENT years the investigation of the magnetic switching behavior of nanostructured permalloy elements [1], [2] has become more advanced due to improvements in numerical micromagnetic methods on the theoretical side and high accuracy fabrication methods, such as electron beam lithography and focused ion beam techniques. The worldwide interest in these elements is their potential for possible future application in high density magnetic data storage and microsensor applications. Shape and magnetocrystalline anisotropy $K$ determine the magnetization reversal properties. In mesoscopic or nanostructured magnets with $K=0$ the switching fields can be varied by the choice of the geometric shape of the magnets and the orientation of the applied field. This phenomenon is described as configurational anisotropy [3]. Numerical micromagnetic modeling using the finite difference or finite element method reveals the correlation between the local arrangement of the magnetic moments and the microstructural features on a length scale of several nanometers and gives a quantitative treatment of the influence of the shape of mesoscopic or nanostructured magnets on the magnetization reversal and switching.

Traditional investigations of magnetization reversal in small ferromagnetic particles assume spherical or ellipsoidal particles uniformly magnetized along the easy direction for zero applied field. At the nucleation field the magnetization starts to deviate from the equilibrium state according to the preferred magnetization mode [4]. The magnetization reversal mechanisms in

Manuscript received October 15, 2000.

This work was supported by the Austrian Science Fund Projects P13260-TEC and Y132-PHY.

The authors are with the Institute of Applied and Technical Physics, Vienna University of Technology, Wiedner Hauptstr. 8-14, A-1040 Wien, Austria (e-mail: fidler@tuwien.ac.at).

Publisher Item Identifier S 0018-9464(01)06171-4. nonellipsoidal particles have been rigorously studied applying finite difference [5], [6] or finite element techniques [7]. The numerical results clearly show that strong stray fields, which cause the magnetization to become inhomogeneously arranged, influence the reversal process drastically [8]-[10].

Differences in switching fields for various directions of the applied magnetic field are predicted from our micromagnetic model calculations for cubic and platelet shaped (squared and triangular) nanoelements neglecting thermally activated magnetization reversal processes and surface anisotropy effects. The influence of the element size on the magnetic switching behavior determined only by strayfield and exchange energy will be shown and possible mechanisms for the magnetization reversal will be discussed.

\section{Micromagnetic Simulation}

\section{A. Micromagnetic and Numerical Background}

Micromagnetism starts from the total magnetic Gibb's free energy, $E$, of a ferromagnetic system, which is the sum of the exchange energy, the Zeeman energy, the magnetostatic energy, and neglecting the magneto-crystalline anisotropy energy [11].

$$
E_{t}(\mathbf{J}, \Psi)=\int\left[\frac{A}{J_{s}^{2}}(\nabla \mathbf{J})^{2}-\mathbf{J} \cdot \mathbf{H}-\mathbf{J} \cdot \nabla \Psi\right] d V
$$

Here $A$ denotes the ferromagnetic exchange constant, $\boldsymbol{J}$ is the magnetic polarization. $\boldsymbol{H}$ denotes the external field. When the components of the polarization vector are approximated by piecewise linear functions on the finite element mesh, the energy functional (1) reduces to an energy function with the nodal values of the vector components as unknowns. Its minimization with respect to the $J_{i}$ at the nodal points, subject to the constraint $|\boldsymbol{J}|=J_{s}$, provides an equilibrium distribution of the polarization. To satisfy the constraint, the polarization is represented by polar coordinates.

The crucial part of the micromagnetic simulation of nanoelements is the accurate calculation of the magnetic stray field. In finite element field calculation, micromagnetic simulations introduce a magnetic scalar or magnetic vector potential to calculate the demagnetizing field. For the calculation of the demagnetizing field of mesoscopic or nanostructured magnets the magnetic scalar potential $\Psi$ was calculated using a hybrid finite element/boundary element technique, which was originally proposed by Fredkin and Koehler [12]. The numerical integration of the Landau Lifshitz-Gilbert equation of motion provides the time resolved magnetization patterns during the reversal process. A Runge-Kutta method optimized for mildly-stiff differential equations [13] proved to be effective 
TABLE I

NUMBER OF SURFACE / VOLUME ELEMENTS

\begin{tabular}{lcccc}
\hline nanoelement & $d=10 \mathrm{~nm}$ & $\mathrm{~d}=20 \mathrm{~nm}$ & $\mathrm{~d}=40 \mathrm{~nm}$ & $\mathrm{~d}=100 \mathrm{~nm}$ \\
\hline Cubic $(\mathrm{c})$ & $192 / 320$ & $192 / 320$ & $768 / 2560$ & $4800 / 40000$ \\
Square $(\mathrm{s})$ & $96 / 80$ & $96 / 80$ & $384 / 640$ & $2240 / 8000$ \\
Triangular $(\mathrm{t})$ & $64 / 67$ & $64 / 67$ & $260 / 455$ & $1264 / 5020$ \\
\hline
\end{tabular}
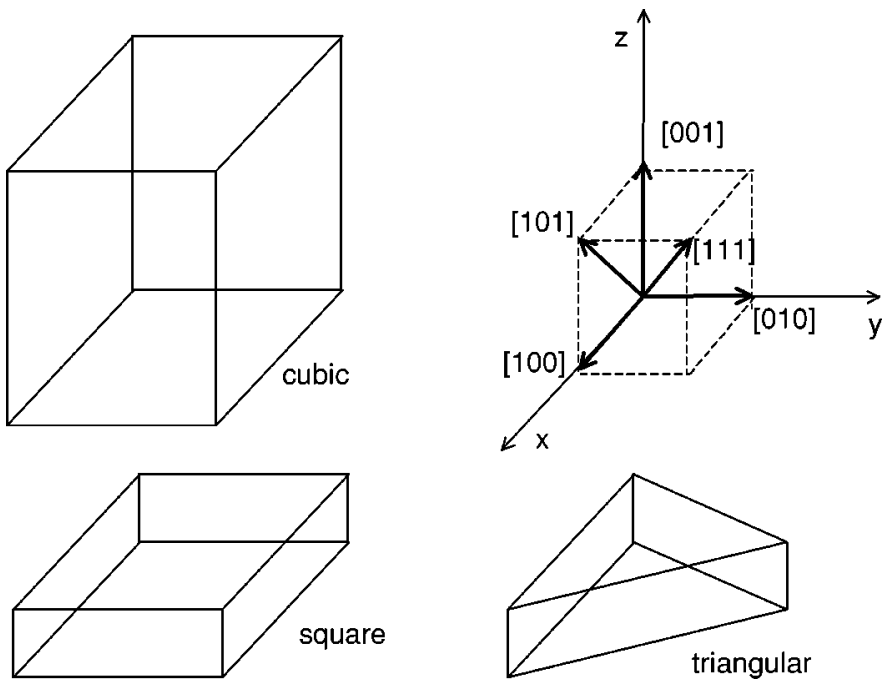

Fig. 1. Schematic drawing of the shape of the simulated elements showing the different applied field directions.

for the simulation using a regular finite element mesh and a Gilbert damping constant $\alpha \geq 0.2$. However, for an irregular mesh as required for triangular nanoelements and $\alpha=0.1 \mathrm{a}$ time step smaller than $10 \mathrm{fs}$ is required to obtain an accurate solution with the Runge-Kutta method. In this highly stiff regime, backward difference schemes allow much larger time steps and thus the required CPU time remains considerably smaller than with the Runge-Kutta method. Since the stiffness arises mainly from the exchange term, the demagnetising field can be treated explicitly and thus is updated after a time interval $\tau$. During the time interval $\tau$ the Gilbert equation is integrated with a fixed demagnetising field using a higher order backward difference method. $\tau$ is taken to be inversely proportional to the maximum torque acting over the finite element mesh.

\section{B. Finite Element Model and Intrinsic Properties}

The nanomagnets were in the size range 10, 20, 40 and $100 \mathrm{~nm}$ for the edge length $d$ and in the thickness range 2, 4, 8 and $20 \mathrm{~nm}$ for the platelet shaped geometries (square and triangular). The aspect ratio between edge length and thickness was kept constant. In order to avoid the influence of the magnetocrystalline anisotropy effects the following material parameters were chosen: $J_{s}=1 \mathrm{~T}, K_{1}=0, A=13 \mathrm{pJ} / \mathrm{m}$ and $\alpha=0.1$

The nanomagnets were discretized into tetrahedral finite elements with a constant edge length of $5 \mathrm{~nm}$ and $2.5 \mathrm{~nm}$ for $10 \mathrm{~nm}$ edge length, respectively. The total number of the elements varied from 88 (10 nm cube) to 44800 (100 nm cube). Table I summarizes the number of surface and volume elements after discretization of the nanoelements.
TABLE II

Calculated Coercive (Switching) Field $\mu_{0} H_{\text {SW }}[T]$

\begin{tabular}{lcccc}
\hline nanoelement & $\mathrm{d}=10 \mathrm{~nm}$ & $\mathrm{~d}=20 \mathrm{~nm}$ & $\mathrm{~d}=40 \mathrm{~nm}$ & $\mathrm{~d}=100 \mathrm{~nm}$ \\
\hline (c) $[001]$ & 0.020 & 0.130 & 0.026 & $0^{*}$ \\
(c) $[101]$ & 0.354 & 0.132 & 0.020 & $0^{*}$ \\
(c) $[111]$ & 0.260 & 0.104 & 0.022 & $0^{*}$ \\
(s) $[001]$ & $0^{*}$ & $0^{*}$ & $0^{*}$ & $0^{*}$ \\
(s) $[010]$ & 0.018 & 0.014 & 0.002 & 0.010 \\
(s) $[110]$ & 0.006 & 0.006 & 0.014 & 0.020 \\
(t) $[001]$ & $0^{*}$ & $0^{*}$ & $0^{*}$ & $0^{*}$ \\
(t) $[010]$ & 0.002 & 0.006 & 0.016 & 0.018 \\
(t) $[100]$ & 0.006 & 0.002 & 0.004 & 0.010 \\
\hline
\end{tabular}

* no switching behavior observed

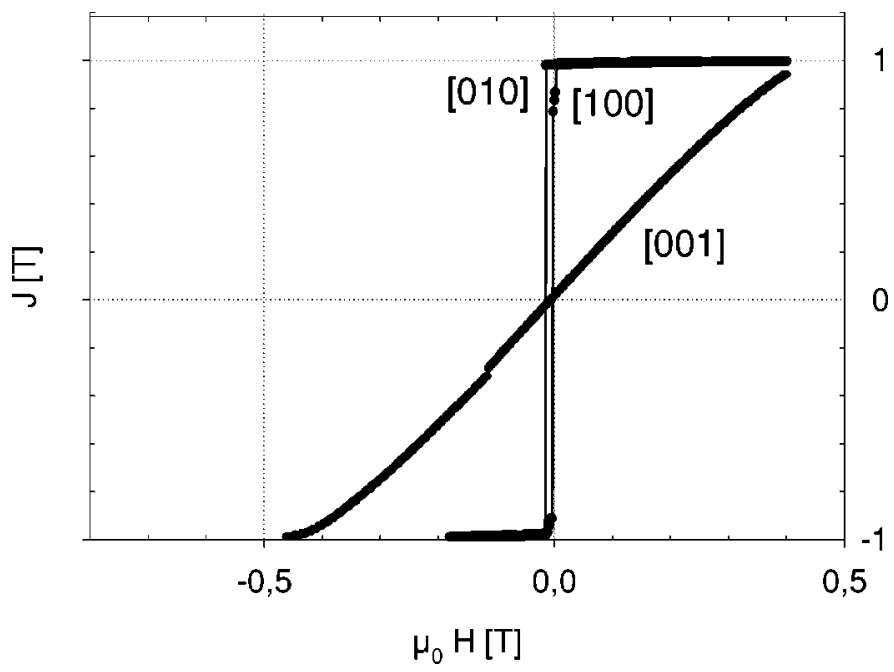

Fig. 2. Numerically calculated demagnetization curves of a triangular platelet with $40 \times 40 \times 8 \mathrm{~nm}^{3}$. The external field was applied parallel to the [001], [010] and [100] directions.

Fig. 1 presents the cubic, squared and triangular nanoelements and shows the magnetic field directions. The magnetic field was applied in certain crystallographic directions, for cubes parallel to [001], [101], [111], for square platelets parallel to [001], [010], [110] and for triangular platelets parallel to [001], [010], [100]. The calculations were started after saturation. The field was reduced in steps of $\mu_{0} \Delta H=0.002 \mathrm{~T}$ starting from $\mu_{0} H=0.4 \mathrm{~T}$.

\section{NUMERICAL RESUlTS}

A 3D micromagnetic FE simulation based on the Landau Lifshitz-Gilbert equation of motion has been used to systematically compare the influence of cubic, cylindrical, disk and platelet (square and triangular) shaped nanoelements on the switching field behavior. Table II shows the dependence of the coercive or switching field with a reversed field applied parallel to the [001], [101] and [111] directions. Depending on the orientation of the field it is clearly visible that a switching behavior occurs for small elements.

Nanomagnets $\geq 40 \mathrm{~nm}$ show an inhomogeneous vortex-like magnetization structure during the reversal process. The resulting switching fields become independent of the direction of the magnetic field. In $100 \mathrm{~nm}$ cubic elements the clear switching characteristic is replaced by inhomogeneous rotation 


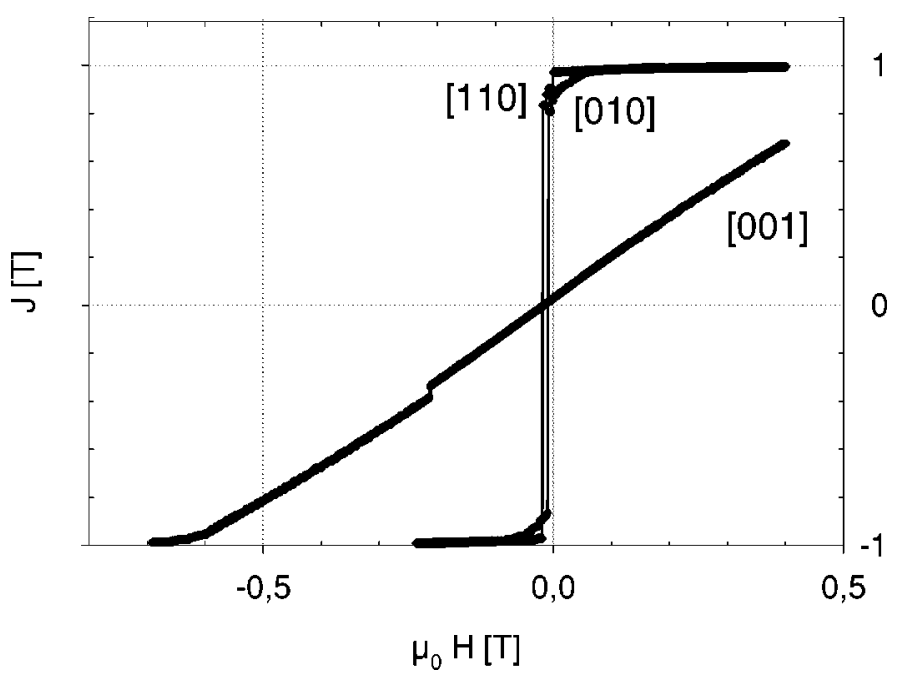

Fig. 3. Numerically calculated demagnetization curves of a square platelet with $100 \times 100 \times 20 \mathrm{~nm}^{3}$. The external field was applied parallel to the [001], [010] and [110] directions.
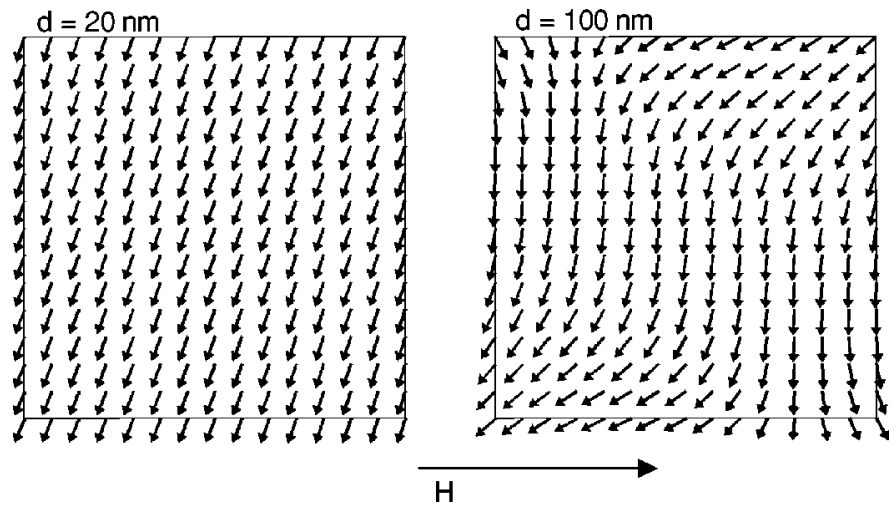

Fig. 4. Comparison of the transient magnetization states during the reversal of square elements of the size $20 \times 20 \times 4 \mathrm{~nm}^{3}$ and $100 \times 100 \times 20 \mathrm{~nm}^{3}$ with zero magnetocrystalline anisotropy under the influence of a constant reversed field of $\mu_{0} H=-0.001 \mathrm{~T}$ parallel to the [010] direction.

of magnetization similar to the "hard direction" rotation. Platelet shaped elements also show this characteristic "hard direction" magnetization reversal perpendicular to the platelet surface.

Only small differences of the switching behavior in various directions within the platelet plane, such as [010], [100] for triangular (Fig. 2) and [010], [110] for square elements (Fig. 3) were found. Increasing the platelet size $\geq 100 \mathrm{~nm}$ the prompt switching changes to an inhomogeneous magnetization reversal resulting in a typical rounded demagnetization curve (Fig. 3). The differences of the magnetization reversal modes are compared in Figs. 4 and 5 for square and triangular elements of different sizes.

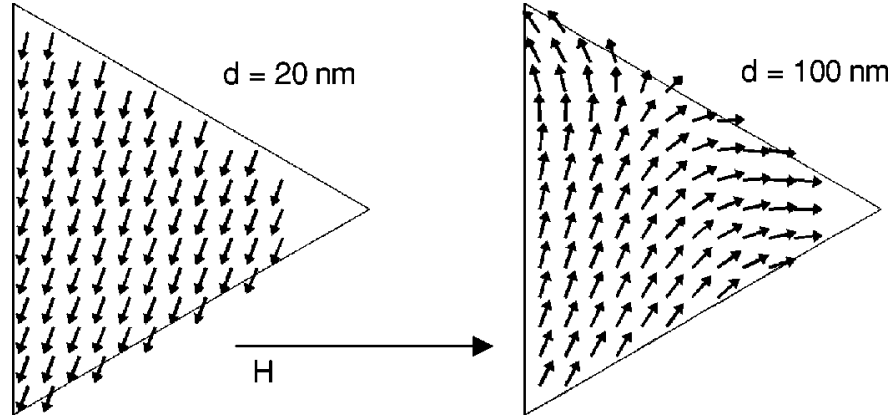

Fig. 5. Comparison of the transient magnetization states during the reversal of triangular elements of the size $20 \times 20 \times 4 \mathrm{~nm}^{3}$ and $100 \times 100 \times 20 \mathrm{~nm}^{3}$ with zero magnetocrystalline anisotropy under the influence of a constant reversed field of $\mu_{0} H=-0.002 \mathrm{~T}$ parallel to the [010] direction.

\section{SUMMARY}

Using the hybrid finite element/boundary element method we investigated the influence of size and shape on the switching dynamics of mesoscopic or nanostructured $\mathrm{Ni}_{80} \mathrm{Fe}_{20}$ elements with $K=0$.

- The numerical results show a strong influence of the element size on the switching behavior.

- Configurational anisotropy effects were only observed in platelet shaped elements with magnetic field directions perpendicular to the platelet plane.

- Inhomogenous magnetization reversal processes become dominant with increasing element size $\geq 100 \mathrm{~nm}$.

\section{REFERENCES}

[1] S. Y. Chou, "Patterned magnetic nanostructures and quantized magnetic disks," IEEE Trans. Magn., vol. 85, pp. 652-671, 1997.

[2] R. D. Gomez, T. V. Luu, A. O. Pak, I. D. Mayergoyz, K. J. Kirk, and J. N. Chapman, "Domain wall motion in micro-sized permalloy elements," $J$. Appl. Phys., vol. 85, pp. 4598-4600, 1999.

[3] R. P. Cowburn, "Property variation with shape in magnetic nanoelements," J. Phys. D, vol. 33, pp. R1-R16, 2000.

[4] A. Aharoni, "Theoretical search for domain nucleation," Rev. Mod. Phys., vol. 34, pp. 227-238, 1962.

[5] M. E. Schabes and H. N. Bertram, "Magnetization processes in ferromagnetic cubes," J. Appl. Phys., vol. 64, pp. 1347-1357, 1988.

[6] Y. Nakatani, Y. Uesaka, and N. Hayashi, "Direct solution of the LandauLifshitz-Gilbert equation for micromagnetics," Jpn. J. Appl. Phys., vol. 28 , pp. 2485-2507, 1989.

[7] T. R. Koehler and D. R Fredkin, "Finite element methods for micromagnetics," IEEE Trans. Magn., vol. 28, pp. 1239-1244, 1992.

[8] R. H. Victora, "Micromagnetic predictions for barium ferrite particles," J. Appl. Phys., vol. 63, pp. 3423-3428, 1988.

[9] Y. D. Yan and E. Della Torre, "Reversal modes in fine particles," J. de Physique, vol. C8, pp. 1813-1814, 1988.

[10] M. E. Schabes, "Micromagnetic theory of nonuniform magnetization processes in magnetic recording particles," J. Magn. Magn. Mater., vol. 95, pp. 249-288, 1991.

[11] J. Fidler and T. Schrefl, "Micromagnetic modeling-The current state of the art," J. Phys. D, vol. 33, pp. R135-R156, 2000.

[12] D. R. Fredkin and T. R. Koehler, "Hybrid method for computing demagnetizing fields," IEEE Trans. Magn., vol. 26, pp. 415-417, 1990.

[13] B. P. Sommeijer, L. F. Shampine, and J. G. Venwer, "RKC: An explicit solver for parabolic PDEs," J. Comp. and Appl. Mathem., vol. 66, pp. 315-326, 1998. 